\title{
A New Method for Detection of Causative Agents at Various Sites of Air Way in Patients with Respiratory Infection
}

\author{
Keizo Matsumoto, Hisao Kimura, Kiyo Nishioka, Yukio \\ Noguchi, Yoshio Uzuka and Ichiyo Honda \\ The First Department of Internal Medicine, Tohoku University \\ School of Medicine, Sendai
}

Matsumoto, K., Kimura, H., Nishioka, K., Noguchi, Y., Uzuka, Y. and HoNDA, I. A New Method for Detection of Causative Agents at Various Sites of Air Way in Patients with Respiratory Infection. Tohoku J. exp. Med., 1974, 112 (4), 383-384 — A new technique for determining causative agents in various sites of air way in patients with respiratory infection was devised in which sputum specimens were directly aspirated from various sites of air way by use of the Kifa-Green catheter and each of them was cultured for the determination of causative agents. This method has an advantage for obtaining information of an exact location of causative agents in air way. Main causative agents detected by this method in patients with various respiratory infection were Haemophilus influenzae, Pseudomonas aeruginosa, and Diplococcus pneumoniae.— respiratory infection; aspirated sputum; small air way; Kifa-Green catheter; quantitative sputum culture

It is desirable to determine the exact location of causative agents in the air way in patients with respiratory infections. For this purpose, in the present study sputum specimens were aspirated from various sites of bronchi of the patients and examined for causative agents.

Methods and patients: The apparatus consists of an outer tube, a Kifa-Green catheter of $2 \mathrm{~mm}$ in outer diameter (normally used for cardiac catheterization) and a Raulrock's syringe (Matsumoto et al. 1970). Two hours before examination, $0.03 \mathrm{~g}$ of codein phosphate is administered to the patient, and 30 minutes before the examination an intramural injection of $50 \mathrm{mg}$ of Hydroxyzine pamoate (Atarax P) is performed as the premedication. After the nasal cavity is anesthetized with $2 \%$ xylocaine and pharynx and larynx are also anesthetized with $4 \%$ xylocaine, the outer tube is passed through the nasal cavity into the trachea and main bronchus. The Kifa-Green catheter is passed through the outer tube and into the bronchus and bronchioles under X-ray control. After confirming that its tip is wedged firmly in the bronchioles gentle negative pressure is applied with the syringe. About $0.5 \mathrm{ml}$ of secretion can constantly be recovered. By this procedure the causative organisms may be isolated from any part of bronchial tree and the total number of bacteria per milliliter can be determined by viable counts.

Patients subjected to this study are out- and in-patients of the First Department of Internal Medicine of the University Hospital, consisting of 112 cases with respiratory infections, such as chronic bronchitis, chronic bronchiolitis, bronchiectasia etc.

Results: All cases that we have ever dealt with are indicated in Table 1.

Causative organisms detected in cases of above diseases are Haemophilus influenzae, Pseudomonas aeruginosa, Escherichia coli and Diplococcus pneumoniae. Above organisms

Received for publication, February 7, 1974. 
TABLE 1. Causative organisms obtained from chronic respiratory infection with the aspiration

\begin{tabular}{|c|c|c|c|c|c|c|}
\hline \multirow{2}{*}{ Clinical diagnosis } & \multirow{2}{*}{$\begin{array}{l}\text { Number of } \\
\text { patients }\end{array}$} & \multirow{2}{*}{$\begin{array}{l}\text { Number of } \\
\text { exp. }\end{array}$} & \multicolumn{4}{|c|}{ Causative organisms } \\
\hline & & & + & & $\geqq 10^{5} \mathrm{ml}$ & - \\
\hline $\mathrm{CPE}$ & 18 & 56 & 24 & 12 & $\left(\begin{array}{l}\text { Str. } 6 \text { Pn. } 4 \text { E. } 3 \text { N. } 3 \\
\text { Sta. H. inf. H. parah. M. }\end{array}\right)$ & 32 \\
\hline Bronchial asthma $+\mathrm{CPE}$ & 2 & 2 & 2 & & & 0 \\
\hline Bronchial asthma & 6 & 12 & 5 & & & 7 \\
\hline Chronic bronchitis & 17 & 36 & 16 & & & 20 \\
\hline Chronic bronchiolitis & 15 & 159 & 109 & 48 & $\left(\begin{array}{l}\text { H. inf. } 13 \text { E. } 7 \text { Str. } 3 \\
\text { Pn. } 2 \text { Sta. } 2 \text { M. } 2 \text { N. } 2 \\
\text { Psend. } 18 \text { (2 patients) }\end{array}\right)$ & 50 \\
\hline Bronchiectasis & 17 & 62 & 38 & 16 & $\left(\begin{array}{l}\text { H. inf. } 8 \text { Str. } 8 \text { N. } 2 \\
\text { Pseud. Sta. M. }\end{array}\right.$ & 24 \\
\hline Pneumonia & 5 & 12 & 6 & 3 & [Sta. Pseud. Str.] & 6 \\
\hline Pulmonary fibrosis & 14 & 41 & 6 & 3 & {$\left[\begin{array}{l}\text { Sta. } 3 \\
\text { Pn. Str. }]\end{array}\right.$} & 35 \\
\hline Pulmonary tuberculosis & 8 & 15 & 9 & 1 & [Str.] & 6 \\
\hline Atypical pneumonia & 2 & 4 & 3 & & & 1 \\
\hline Normal & 1 & 1 & 0 & & & 1 \\
\hline Other diseases & 7 & 16 & 5 & 1 & [Str.] & 11 \\
\hline Total & 112 & 416 & 223 & & 84 & 193 \\
\hline
\end{tabular}

E.: E. coli; H. inf.: Haemophilus influenzae; H. parah.: Haemophilus parahemoliticus; M.: Micrococcus; N.: Neisseria; Pn.: Diplococcus pneumoniae; Pseud.: Pseudomonas aeruginosa; Sta.: Staphylococcus; Str.: a-Streptococcus. Observation period: 1967.1.1-1973. 6.30.

are isolated over $10^{7}$ per milliliter in one part of bronchus by the quantitative sputum culture. But normal floras like Micrococcus or Neisseria catarrhalis are also isolated over $10^{7}$ per milliliter in mixture of two or three organisms in some cases. So there is room for discussion about the pathogenisity of these normal floras. None of the above mentioned microorganisms is detected by this method in the sputum from individuals free from respiratory infection.

Since detected agents are often different in the right and left bronchus or in each bronchus, causative agents must be considered to be present isolating in each bronchus. Precise mode of respiratory infection can be analyzed by scrutinizing the correlation between each microorganism which is isolated from various sites of respiratory tract by this method.

Advantage of this method: The inserted tip of the eatheter is clearly demonstrated under the X-ray control by the use of Kifa catheter. Several catheters can be inserted to the same patient in one examination. The outer diameter of the catheter $(2 \mathrm{~mm})$ is so small enough that the investigation of so called small airway is easily performed. By the series of these examinations the explanation of correlation between the change of causative organisms and chemotherapy is easily demonstrated.

Side effect during this procedure: The following two troubles have been noted in two cases during this procedure. Blood streaked sputum was obtained in one case which was probably due to abrasion by the inserted catheter. Spontaneous pneumothorax developed in one early case, which might be caused by the change of position leaving the catheter wedged and could be avoided by getting the inserted catheter free in the bronchus.

In conclusion, this method is less hazardous and very useful not only for the decision of causative agents but also for the analysis of the mode of variable respiratory infections.

\section{Reference}

1) Matsumoto, K., Yokoyama, K., Nakamura, T. (1970) Chemotherapy of chronic respiratory infections using Cephaloridine. Postgrad. med. J., 10, 123-128. 